\title{
LA POLÍTICA PÚBLICA DE SALUD A NIVEL LOCAL. ANÁLISIS DE LA TRANSFORMACIÓN DEL CENTRO DE SALUd FAMILIAR ISABEL JIMÉNEZ RIQUELME DE Tirúa, Biobío, Chile
}

\author{
Ninette Sepúlveda Alecoi \\ ninettesepulveda@gmail.com \\ Universidad Alberto Hurtado
}

El artículo analiza el proceso de implementación del Centro de Salud Familiar Isabel Jiménez Riquelme ubicado en la VIII región de Chile. A partir de entrevistas a usuarios y funcionarios se evalúa la implementación de la política pública de salud para la localidad de Tirúa, entre los años 2006 y 2011. Se concluye que se han realizado acciones que reflejan un "modelo" de salud caracterizado por una mezcla de lo biomédico con pequeños matices que integran lo bio-psicosocial, el enfoque familiar, y un enfoque intercultural (aplicado a la medicina mapuche). Se constata la coexistencia de otros modelos médicos en las prácticas de los usuarios, adscritas a medicinas populares, indígenas y de autoatención, con una fuerte presencia de la dimensión espiritual o religiosa.

Palabras clave: Politicas públicas, salud, participación. 


\section{ANALYSIS OF THE IMPLEMENTATION OF HEALTH POLICIES: THE CASE OF THE ISABEL JIMÉNEZ RIQUELME FAmily HeAlth CENTRE in TIRÚA, BÍOBIO,CHILE}

This paper analyzes the transformation process of the Isabel Jiménez Riquelme Family Health Centre, located in Chile's VIII region. Through interviews of users and officials, the implementation of public health policies in the town of Tirúa during the period of 2006 and 2011 are evaluated. We conclude that actions taken reflect a "model" of health characterized by a mixture of the biomedical together with elements of the bio-psychosocial, a family-oriented approach, as well as an intercultural approach (applied to Mapuche medicine). We confirm the coexistence of other medical models related to folk medicine, indigenous and self care, with a strong presence of the spiritual or religious dimension.

Keywords: Public policies, health, participation. 


\section{INTRODUCCIÓN}

El presente artículo pretende entregar un análisis de la implementación de la política pública de salud, a partir de los resultados de un estudio previo realizado por su autora. Este artículo forma parte de una investigación cualitativa titulada: "De Consultorio a Centro de Salud Familiar, la voz de los actores": Estudio de Caso del CESFAM Isabel Jiménez Riquelme de Tirúa, 2006-2011. Es un trabajo exploratorio en el cual se utilizaron las técnicas como la entrevista semi estructurada y el análisis documental para la recolección de información y el análisis de contenido como método para el trabajo de los discursos generados a partir de dichas técnicas. Su relevancia está dada por destacar la percepción de los propios actores involucrados, es decir usuarios(as) y funcionarios(as) que en el día a día se enfrentan a las acciones y cambios derivados de la transformación del consultorio a CESFAM.

Los resultados de este estudio, dan a conocer el proceso de transformación del CESFAM a partir de la percepción que tienen los principales actores involucrados, su visión de cambio, las disonancias existentes entre ellos y un análisis de la pertinencia de esta política de salud en su proceso de implementación. Con ello, se espera que este estudio permita mejorar la ejecución y pertinencia de las estrategias de salud en el ámbito local y contribuir al conocimiento y análisis de la aplicación de las políticas públicas de salud que surgen desde el nivel central. El problema de investigación está enfocado a conocer el proceso de transformación a nuevo modelo de salud llevado a cabo por el actual CESFAM I.J.R. en Tirúa a partir de la percepción de funcionarios y usuarios. Este proceso trae consigo una serie de cambios en el funcionamiento a nivel local de tipo administrativo, financiero, técnico y de relación entre usuarios (comunidad) y equipo de salud que para esta investigación son trascendentales y necesarios de conocer y analizar.

Nadler (1980, citado en Ringeling, 1986: 200) plantea que la introducción de cualquier modificación trae consigo grandes problemas como la resistencia al cambio, los conflictos de poder, la alteración del control organizacional y su estabilidad. Así también lo avala Kisil (1984, citado en Ringeling, 1986:200), al señalar que el modo de entender el proceso salud - enfermedad en el enfoque familiar revitaliza y re-humaniza la medicina del nivel primario, pero como toda 
innovación es potencialmente generadora de conflictos, ya que entra en competencia con otros intereses a veces contrapuestos. Esta situación de conflicto y tensión está dada por un planteamiento dicotómico referido a la confrontación de los paradigmas biomédico y el biopsicosocial en el accionar de los establecimientos de salud primaria. Por tanto, en este escenario se contraponen, el sistema de atención de salud oficial, centrado en el usuario como individuo, con acciones de salud focalizadas en lo curativo, carentes de un trabajo en equipo; frente a la evidencia de un cambio socio-demográfico y epidemiológico de la población, que demanda una mirada distinta, con un modelo de atención familiar más moderno, cercano a las necesidades de la población, con énfasis en la estructura familiar, en su comunidad, con un fuerte trabajo comunitario y una red intersectorial diversa (Cortés, Lerma \& Araya, 2004).

Por su parte, Menéndez (2010) usa el concepto de Modelo Médico Hegemónico pues se tiende a establecer una relación de hegemonía - subalternidad de la biomedicina respecto de otras formas de atención no biomédicas, que quedarán normalmente excluidas, ignoradas, o estigmatizadas, aunque podrán optar a una aceptación crítica, o a una apropiación de uso complementario, pero siempre de carácter subordinado.

Esta situación de conflicto, tensión, dicotomía, hegemonía-subalternidad referida por los autores citados, se vivencia en la práctica cotidiana, pero no siempre es un ejercicio analítico consciente por parte de los actores sociales involucrados. Agregan Cuevas, López \& Mancilla (2006) que en esta confrontación de modelos, se desconoce los discursos que los profesionales de la atención primaria de salud tienen en relación a estos enfoques y cómo estos coexisten en esta práctica diaria. Para el caso de Tirúa, no sólo se desconocen los discursos de los profesionales, sino también las percepciones de todos los funcionarios, usuarios, dirigentes y actores vinculados que vivencian en la práctica este fenómeno, pero que no se realiza un registro de manera sistemática, ni investigaciones cualitativas al respecto. Bajo estos argumentos, se fundamenta esta investigación con el fin de obtener elementos de análisis y reflexión para mejorar las políticas locales de salud en Tirúa. 
La hipótesis central supone que el proceso de transformación que ha vivido el CESFAM I.J.R. de Tirúa, que incluye enfoques de salud integrales con enfoque familiar, comunitario y con pertinencia cultural, se da en una situación de tensión y confrontación en el quehacer dada por la subyacente hegemonía del modelo médico por sobre los otros enfoques y formas de atención. Las hipótesis secundarias plantean que: El modelo de política pública obedece al estilo "Top Down", pues no existe retroalimentación directa entre las etapas de formulación e implementación; existe disonancia entre la percepción de funcionarios y usuarios sobre el proceso de transformación a CESFAM en la comuna de Tirúa y que existe en funcionarios y usuarios una percepción de cambios en el sistema de atención a raíz del proceso de transformación a CESFAM en Tirúa. Por tanto, el objetivo general es describir, analizar y discutir el proceso de transformación de la atención primaria en el CESFAM I.J.R. de la comuna de Tirúa, Región del Biobío, entre los años 2006 - 2011. Para su logro se plantean tres objetivos específicos que dicen relación con describir el proceso de transformación de consultorio a centro de salud familiar que propone el MINSAL para la atención primaria; describir y analizar el proceso de implementación del CESFAM I.J.R. llevado a cabo en la comuna de Tirúa a partir de la percepción de usuarios y funcionarios y analizar y discutir la pertinencia de la aplicación en el nivel local de esta política pública de salud diseñada desde el nivel central.

\section{i. Antecedentes Teóricos y/o Conceptuales}

El encuadre teórico está enfocado al ámbito político administrativo centrado en el concepto de política pública y el ámbito de la salud referido al concepto de modelos médicos e interculturalidad en salud. En primer lugar, las políticas públicas son "el conjunto de objetivos, decisiones y acciones que lleva a cabo un gobierno para solucionar los problemas que en un momento determinado los ciudadanos y el propio gobierno consideran prioritarios" (Tamayo, 1997:281).

Según Tamayo (1997), las políticas públicas se pueden entender como un proceso que se inicia cuando un gobierno o un directivo público detecta la existencia de 
un problema que por su importancia merece su atención y termina con la evaluación de los resultados que han tenido las acciones emprendidas para eliminar, mitigar o variar ese problema.

Esta investigación intenta descubrir cómo se da el proceso en la práctica y centra su análisis en la etapa de implementación, la cual corresponde a "la fase de una política pública durante la cual se generan actos y efectos a partir de un marco normativo de intenciones, de textos, o de discursos", es decir, el espacio que existe entre la política como declaración de intenciones y la política como declaración de resultados, y por tanto, el conjunto de acciones que transforman las intenciones en resultados observables" (Blázquez y Morata, 2005:153).

Blázquez y Morata (2005) señalan que el análisis de la implementación a partir de los años 70 se concentró en tres modelos:

- Modelo Top-Down: "se presta poca atención al vínculo que existe entre la formulación-implementación-redefinición, por tanto, no se busca explicar cómo las decisiones políticas se transforman en servicios públicos, pues la orden sobre la cual debía ser la actividad desplegada por los aparatos administrativos se realiza sin ninguna interferencia en el camino" (Blázquez y Morata, 2005: 156).

- Modelo Buttom-Up: surge tras la incapacidad del anterior modelo para dar explicación a los fracasos de las políticas públicas. "En este modelo, el proceso ya no será lineal ni unidireccional, sino más bien circular, donde caben las relaciones recíprocas de poder y negociación (...) La ejecución por tanto tiene una dinámica propia y no es una mera puesta en práctica de las decisiones a través de la obediencia" (Blázquez \& Morata, 2005:161).

- Una tercera generación de estudios, postula la no necesaria elección entre un modelo y otro, "Top Down" frente a "Buttom-Up", sino que ambos podrían ser aplicados en la explicación del proceso de implementación, es decir, "deberían integrar el macro mundo de los decisores con el micro mundo de los implementadores individuales" (Blázquez \& Morata, 2005: 163). 
En segundo lugar, la investigación se sitúa en el concepto de modelos médicos, planteado por Menéndez (1982:3), para quien modelos médicos son "aquella construcción que a partir de determinados rasgos estructurales supone no sólo la producción teórica, técnica, ideológica, social y económico-política de los médicos, sino también la de los conjuntos implicados en su funcionamiento". Otra definición complementaria, que agrega dos temas de mucha importancia para esta investigación: el de agentes médicos y prácticas médicas, plantea que "el modelo médico se refiere al ámbito ideológico, más tradicionalmente vinculado con la cultura y que abarca conceptos y definiciones, en las que la salud y enfermedad se entrecruzan con la religión, de la concepción de la vida y de la muerte, de la enfermedad, el vínculo entre el hombre y los medios natural y sobrenatural, del cuerpo, etc.” (Citarella, 2010:79).

Los agentes médicos son todos aquellos que realizan las prácticas de salud. Cada tipología de agente médico tiene su propia estructura organizacional, sus prácticas, saberes y concepciones. Análogamente, existen diferentes niveles de especialización, diferencias entre agentes, roles y funciones creativas". Por su parte, las prácticas médicas son una serie de acciones que se realizan en determinado momento (consulta, diagnóstico, hospitalización, tratamiento, entre otros), que han dado lugar a reglas que permiten a las instituciones sanitarias establecer criterios de conductas o protocolos más o menos homogéneos para resolver problemas de salud. Dentro de estas prácticas son muy relevantes los espacios y los escenarios, en los cuales se desenvuelve la acción médica, así como el sistema de códigos de lenguaje, un sistema de comunicación médico-paciente característico de cada uno de los esquemas médicos (Citarella, 2010). Por su parte, Menéndez (1982) plantea una clasificación útil para los objetivos de investigación que consiste en tres modelos: Modelo Médico Hegemónico, Modelo Médico Alternativo Subordinado y Modelo Médico de Autoatención.

El Modelo Médico Hegemónico (MMH), es el "conjunto de prácticas, saberes y teorías generados por el desarrollo de lo que se conoce como medicina científica, el cual desde fines del siglo XVIII ha ido logrando establecer como subalternas al conjunto de prácticas, saberes e ideologías teóricas hasta entonces 
dominantes en los conjuntos sociales, hasta lograr identificarse como la única forma de atender la enfermedad legitimada tanto por criterios científicos, como por el Estado" (Menéndez, 1988:1). Según Citarella (2010) este modelo es aquel que se despliega principalmente en el sector público (hospitales, cajas de salud, postas de salud públicas) y en el sector privado (hospitales, clínicas privadas y ONG), así como el sector individual privado (consultorios privados). Sus principales rasgos estructurales son el biologisismo, la homologación, el individualismo, el marco teórico evolucionista-positivista, la mercantilización de la salud, la relación asimétrica entre médicos y pacientes. Así: "el conjunto de características y las funciones del MMH y no cada característica en sí misma tiende a establecer una relación de hegemonía-subalternidad de la biomedicina respecto de otras formas de atención no biomédicas, que quedarán normalmente excluidas, ignoradas, o estigmatizadas, aunque podrán optar a una aceptación crítica, o a una apropiación de uso complementario, pero siempre de carácter subordinado" (Menéndez, 2010:105).

El Modelo Médico Alternativo Subordinado (MMAS), según Menéndez (1982) es aquel donde se integran las prácticas reconocidas generalmente como "tradicionales", pero en gran medida son derivaciones o emergencias del $\mathrm{MMH}$ que sintetizan y transforman inclusive a las llamadas prácticas tradicionales. En otras palabras, agrupa las prácticas de los "curanderos", la medicina ayurvédica, de la acupuntura, de la herbolaria, de las técnicas basadas en la eficacia simbólica; pero también incorpora a las técnicas espiritistas, a la quiropraxia, al desarrollo de las técnicas psicoterapeutas y socio-terapéuticas "tipo grupos de encuentro" y "potencial humano", a los curadores espirituales de masa, a las prácticas tipo culto del peyotl, a las "técnicas" tipo dianética, piramidales, etc., a las técnicas naturistas, a las "clínicas del cuerpo", tanto para tratamientos de belleza, dietéticos, como sexuales, etc.

Citarella (2010) en tanto, señala que la estructura de este modelo es más compleja que el MMH por cuanto refleja la diversidad cultural de cada país o región. En otras palabras, existe una concepción abierta, en la que el funcionamiento del cuerpo no está relacionado únicamente con los fenómenos biológicos, fisiológicos, y bioquímicos, sino que está vinculado al ámbito espiritual, religioso, ecológico y social, y está abierto a la influencia de fuerzas externas que pueden atacarlo. 
El Modelo Médico de Autoatención (MMA) corresponde a las "representaciones y prácticas que la población utiliza a nivel de individuo o grupo para diagnosticar, explicar, atender, controlar, aliviar, aguantar, curar, solucionar o prevenir los procesos que afectan la salud en términos reales o imaginarios, sin la intervención central, directa e intencional de curadores profesionales aun cuando estos puedan ser de la referencia de la actividad de autoatención. Así la autoatención implica decidir la autoprescripción y el uso de un tratamiento autónomo o relativamente autónomo" (Menéndez, 2010:106). Destaca el autor, que esta autoatención puede suponer desde acciones conscientes de "no hacer nada" pero encaminadas a la cura, hasta el uso de equipos de determinado grado de complejidad. Este modelo es estructural a toda sociedad, suponiendo el real primer nivel de atención de la misma. Dicha estructuralidad se da a partir de las funciones sociales, económicas, ideológicas y técnicas que cumple para los grupos en los cuales opera (Menéndez, 1982).

En el año 2010, Menéndez agrega que la autoatención puede ser analizada en dos niveles, uno amplio y otro restringido. El primer nivel hace referencia a todas las formas de autoatención que se requieren para asegurar la producción biosocial de los individuos y grupos a nivel de los microgrupos y especialmente del grupo doméstico. La definición restringida se refiere a las representaciones y prácticas aplicadas intencionalmente al proceso de salud-enfermedad-atención, por ejemplo, la crianza de los hijos, supone aplicar saberes a toda una serie de aspectos como amamantamiento, otros tipos de alimentación, aseo, control de esfínteres, formas de descanso incluidas las horas y tiempos de sueño. Citarella (2010) agrega que se adscriben a la cultura y medicina popular una multitud extraordinariamente heterogénea de modelos culturales y prácticas de referencias que en América Latina se relacionan con procesos de sincretismo entre la tradición hispánica y la indígena.

$\mathrm{Al}$ reconocer distintos modelos médicos en el área de la salud es posible asociar el concepto de intercultural. Este concepto fue acuñado en el siglo pasado por las ciencias sociales, especialmente por la antropología, para denotar las relaciones que se establecen entre dos o más culturas (Campos, 2010). Ahora bien, la salud intercultural es como la "práctica y el proceso relacional que se establece entre el personal de salud (médicos, enfermeras, etc.) y los pacientes (y sus familiares) en el que ambas partes pertenecen a culturas diferentes y en el que se requiere un en- 
tendimiento recíproco para que el resultado del contacto (consulta, intervención, orientación) sea satisfactorio para ambas partes" (Campos, 2010:35).

En Chile el modelo intercultural en salud "busca provocar un acercamiento entre paradigmas, un encuentro de distintas formas de entender y enfrentar el proceso de salud-enfermedad: lo oficial y lo indígena bajo el concepto de interculturalidad" (Sáez, 2007: 163).

\section{Antecedentes Empíricos y/o Contextuales}

Existen algunos hechos relevantes que ayudan a contextualizar el tema de investigación y que corresponden a hitos del quehacer de la salud a nivel internacional, nacional y local que influyen en el estado del arte de esta investigación. A nivel internacional, destaca la Conferencia Internacional sobre Atención Primaria en Salud, celebrada en 1978 en Alma Ata; la Primera Conferencia Internacional de Promoción de la Salud, realizada en Ottawa en 1986; el Convenio 169 sobre Pueblos Indígenas y Tribales de Países Independientes de la OIT en el año 1989; Iniciativa de Salud de los Pueblos Indígenas de las Américas (SAPIA) de la OPS en 1993 y la Declaración Universal de Derechos de los Pueblos Indígenas de la ONU en el 2007.

En el plano nacional, se puede mencionar algunos hechos relevantes que comienzan en la década del noventa con los procesos de cambios políticos. Se estableció una nueva política de salud basada en los principios de equidad, eficiencia social, participación social, respeto por la dignidad de las personas y solidaridad, a raíz de la cual se incorpora el "Modelo de Atención Integral con Enfoque Familiar y Comunitario", que surge como respuesta a los cambios culturales, sociales del perfil epidemiológico ocurridos en Chile. Este "modelo" buscaba transformar los consultorios dado su limitado abordaje de la salud, a la concepción biomédica. Por tanto, se incorporó en primer lugar el concepto de "centro de salud" y posteriormente, se promovió que los centros de salud debían convertirse en "centros de salud familiar". 
En el año 2000 surge con fuerza la idea de participación en salud, y con ello la puesta en marcha de la reforma sectorial de salud. Para el caso de atención primaria la reforma no se expresó a nivel legal, pero sí a nivel político un compromiso presidencial de aumentar significativamente el financiamiento de la atención primaria, pasando del $12 \%$ al $30 \%$ de los recursos públicos a la salud. Se incluye la implementación de un "modelo de salud familiar", sobre la base de equipos de cabecera que se hacen responsables de un número determinado de familias del sector, de seguimiento permanente (Drago, 2006).

Paralelamente a todos estos cambios, se realiza el "Primer Encuentro Nacional de Salud y Pueblos Indígenas" realizado en la Región de la Araucanía en 1996, el cual generó las bases para la creación del Programa de Salud y Pueblos Indígenas en 1996 y en el año 2001 el Programa de Desarrollo Integral de Comunidades Indígenas (Orígenes) en Convenio BID/MIDEPLAN enfocado a sectores rurales con alta concentración de población indígena. Posteriormente, en el año 2003 se crea la Política de Salud con Pueblos Indígenas, cuyo fundamento de base era el reconocimiento de que ningún sistema médico es capaz de satisfacer por sí solo todas las demandas de salud que presenta una población. El propósito de esta política es contribuir al mejoramiento de la situación de salud de los pueblos originarios, a través del desarrollo de un modelo de salud intercultural que involucre la participación en la construcción, ejecución, control y evaluación del proceso (MINSAL, 2006).

\section{Marco metodolóGico}

La metodología utilizada es de tipo cualitativo, se enmarca en el paradigma constructivista y es denominada también hermenéutica o interpretativa según la clasificación de Guba \& Lincoln (ver Ruiz, 2003:58). Para llevar a cabo los objetivos específicos se analizaron documentos oficiales del Ministerio de Salud. Además se aplicaron y analizaron entrevistas semiestructuradas a funcionarios de salud y usuarios del CESFAM, lo cual se complementó con el análisis de documentos institucionales del establecimiento, como libros de actas del equipo gestor y sectores e informes de trabajo. 
El método utilizado en esta investigación correspondió al estudio de caso, es decir, se llevó a cabo un "proceso de indagación caracterizado por un examen detallado, comprehensivo sistemático y en profundidad del objeto de interés" (Rodríguez, et al., 1999:92). El estudio desarrollado es de tipo exploratorio y el tipo de muestreo utilizado según la clasificación de Ruiz (2003) es de tipo intencional-teórico, puesto que las unidades de muestreo se establecieron en base a los perfiles definidos conceptual y previamente. Estas son: discursos de funcionarios del CESFAM I.J.R., hombres y mujeres, discursos de dirigentes sociales en vinculación con el CESFAM, Postas o CECOSF, discursos de usuarios del CESFAM, Postas, CECOSF que hayan recibido algún tipo de atención de salud, documentos de planificación y quehacer del CESFAM que den cuenta del proceso investigativo y otros documentos de trabajo del MINSAL, OMS, OPS y OIT. Se utilizaron dos técnicas de recolección: entrevista individual semi-estructurada (a 21 funcionarios y 24 usuarios) y documentos institucionales (en total 17).

Las dimensiones de estudio y guías para la recogida de datos son: organización, atención, participación, intersectorialidad y pertinencia cultural. La estrategia de análisis utilizada en esta investigación es la propuesta por Miles y Huberman (ver Rodríguez, et al:: 204) que contempla tres momentos: reducción de datos (separación en unidades, identificación y clasificación de unidades, síntesis y agrupamiento); disposición y transformación de datos; y obtención y verificación de conclusiones (obtención de resultados y conclusiones, verificación de conclusiones). En el proceso investigativo se utilizó el software MAX QDA, el cual permitió reducir la información para llegar a las conclusiones generales. 


\section{AnÁlisis de los REsultados}

Este capítulo se presenta en base al contraste de la hipótesis principal e hipótesis secundarias, con el trabajo de campo. Para ello, se realizará, en primer lugar, una breve presentación de lo que ha implicado el proceso de implementación del CESFAM I.J.R. en la comuna de Tirúa en relación con lo planteado por el MINSAL. Posteriormente, se analizará cada hipótesis utilizando cuadros explicativos que se expondrán en el siguiente orden: percepción de cambios de usuarios y funcionarios en cuanto al proceso de implementación, disonancias entre funcionarios y usuarios, estilo de la política pública y finalmente, el reconocimiento y relación entre los modelos médicos.

\section{El PRoceso de IMPLEMENTACión DEL CESFAM I.J.R.}

Este proceso se realiza en base a cinco dimensiones establecidas por el MINSAL. En todas ellas se han realizado acciones en torno a las directrices estipuladas, sin embargo, se aprecian diferencias en cuanto a los tipos, énfasis, cantidad de actividades y en cuanto a los actores participantes, sectores y postas de salud. Esta descripción se refleja en lo siguiente: 


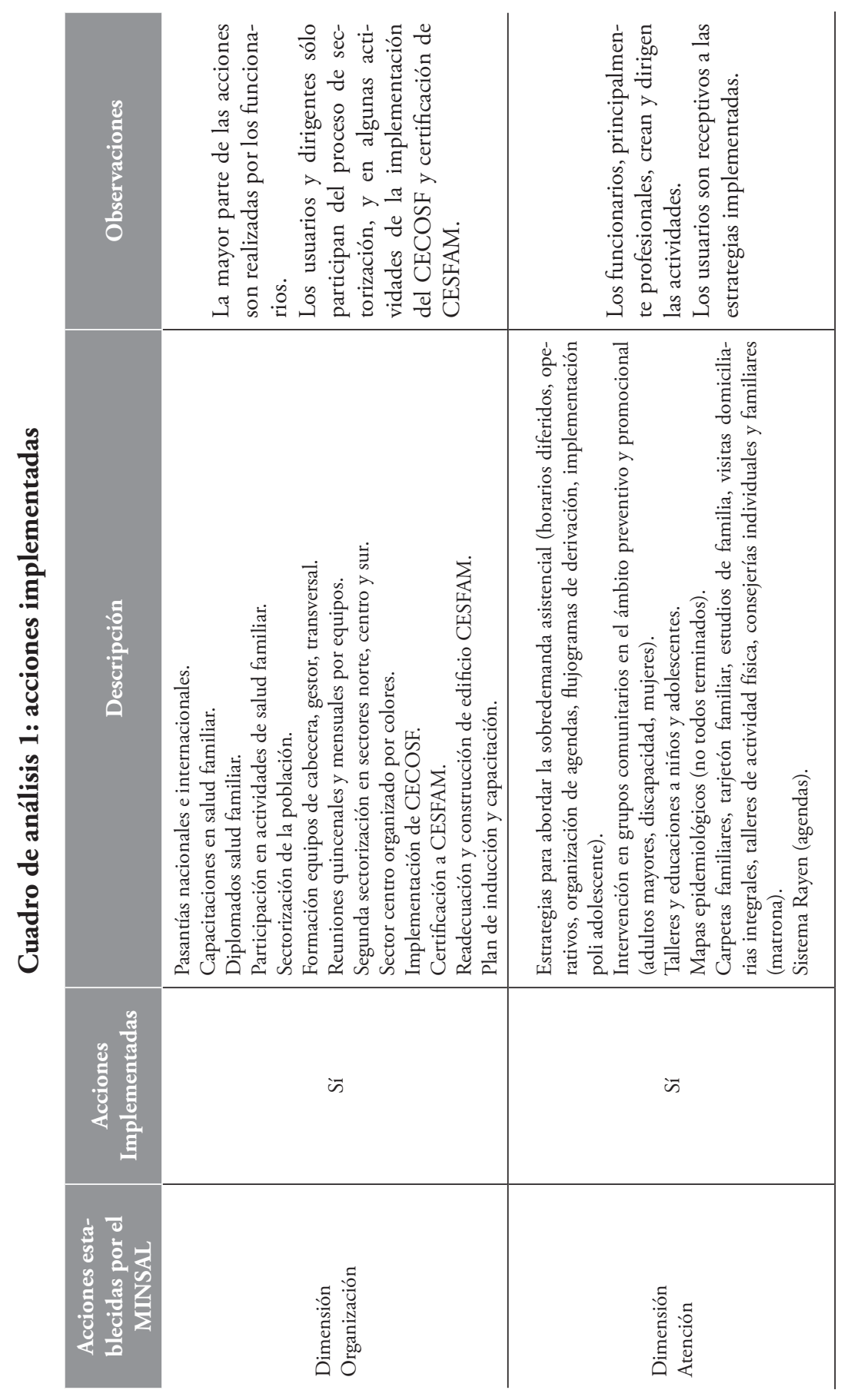




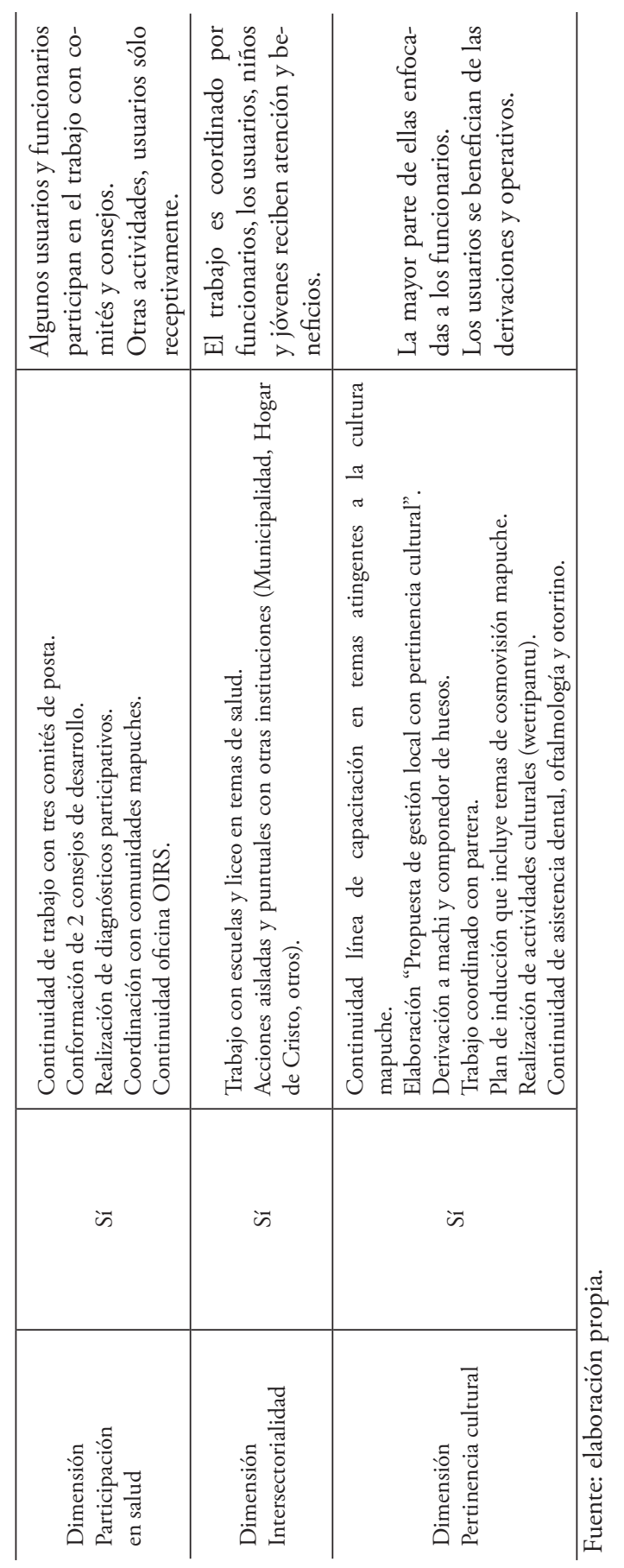


El cuadro muestra que las dimensiones atención y organización, son los espacios en donde se han incorporado más y nuevas estrategias de salud, las cuales han sido creadas y dirigidas principalmente por funcionarios, en especial, por el grupo de profesionales. Entre ellas destacan la sectorización, conformación de equipos, actividades de prevención y promoción, estudios de familia, tarjetones familiares, capacitaciones, entre otros, que han marcado la distinción con respecto a la organización del "antiguo consultorio".

A partir de lo anterior, es interesante hace un primer análisis sobre lo que propone el MINSAL para el proceso de transformación a CESFAM y las actividades o estrategias efectivamente incorporadas por este establecimiento en la comuna de Tirúa. Al respecto, se puede señalar que existe una clara intención a partir del año 2003, de las respectivas jefaturas del CESFAM por llevar a la práctica las estrategias encomendadas, esto se constata en las sucesivas acreditaciones que le ha tocado presentar. A ello, se suma la voluntad de la mayor parte de los funcionarios en crear, liderar y ejecutar las actividades, movilizando recursos humanos, materiales e institucionales y también la apertura y disposición de líderes, dirigentes, la comunidad organizada y algunas otras instituciones para concretar avances. $\mathrm{Al}$ mismo tiempo, el trabajo de campo constató que el proceso no ha sido fácil, ya que en la práctica hay acciones que se realizan con dificultad o que son imposibles de realizar por obstaculizadores que surgen del ámbito institucional, comunal y provincial. Estos obstaculizadores tienen que ver con la escasez de recursos materiales, de infraestructura y humanos, las distintas y a veces contrapuestas visiones de los funcionarios, así también, la dispersión geográfica, la escasa conectividad en medios de transporte, la deficiente comunicación con los usuarios y comunidades y la dificultad para cumplir sus expectativas. Esta situación deja en claro que la implementación no es automática, ni se aplica de manera textual, sino que queda expuesta a un contexto especial donde se conjugan factores políticos, sociales, económicos y culturales.

\section{Percepción de CAMBios}

La percepción de los cambios en el quehacer del CESFAM a raíz del proceso de transformación, se categorizó en tres niveles. El primero, cambios trascendentales que aluden a un proceso profundo y determinante en todas las dimensiones 
del quehacer del establecimiento; el segundo, cambios parciales que implican transformaciones sólo en algunos ámbitos y dimensiones, pero no permiten hablar de modificaciones relevantes; finalmente, los cambios minimos entendidos como transformaciones muy específicas y poco visibles. Al respecto, tanto usuarios como funcionarios sólo reconocen cambios parciales y mínimos tal como se aprecia en el siguiente cuadro:

\section{Cuadro 2: Percepción de cambios}

\begin{tabular}{l|c|c}
\multicolumn{1}{c|}{ Cambios } & Funcionarios & Usuarios \\
Trascendentales & No & No \\
\hline Parciales & Sí \\
& $\begin{array}{c}\text { Organización, Participación, } \\
\text { Atención, Pertinencia cultural }\end{array}$ & Organización \\
\hline Mínimos & Intersectorialidad & Sí \\
& Pertinencia cultural, Intersectorialidad \\
\hline
\end{tabular}

Fuente: elaboración propia.

Este cuadro permite confrontar la primera hipótesis secundaria planteada en el trabajo de investigación, la cual plantea que "existe en funcionarios y usuarios una percepción de cambios en el sistema de atención a raiz del proceso de transformación a CESFAM en la comuna de Tirúa”. Al respecto, el trabajo investigativo no permite rechazar ni confirmar completamente dicha hipótesis, puesto que, si bien, se perciben por parte de los grupos entrevistados cambios parciales o mínimos en algunas dimensiones, los informantes no revelan cambios trascendentales positivos para el mejoramiento del quehacer del CESFAM.

En primer lugar, la totalidad de los cambios advertidos por los usuarios, denominados "parciales" en el cuadro No 2, están dentro de la dimensión organización y corresponden al proceso de sectorización y conformación de equipos de cabecera, infraestructura y recursos materiales. Destaca positivamente el nuevo CECOSF de Quidico y aquellas postas que han implementado proyectos de mejoramiento de infraestructura, también aparecen las expectativas en torno al nuevo CESFAM de que el nuevo edificio solucione gran parte de las falencias en la atención. Esto 
permite señalar que para los usuarios, resulta más fácil percibir cambios tangibles que ideas abstractas como "modelos" o "enfoques" de salud.

En segundo lugar, hay algunos usuarios que notan cambios "mínimos" en cuanto a la atención, participación en salud, pertinencia cultural e intersectorialidad, pues distinguen algunas acciones aisladas y en general sólo aquellos que se han dedicado a realizar alguna labor de dirigente o a participar de algunas de las organizaciones de salud. Estas estrategias tienen que ver con la realización de visitas domiciliarias, actividades de promoción con niños, adolescentes, mujeres y adultos mayores; conformación e integración de algunos usuarios y dirigentes a los comités y consejos de desarrollo, acceso a diagnósticos y tratamientos de machi, componedor de huesos y partera, operativos de especialidad para comunidades mapuches; y trabajos con los establecimientos educacionales de la comuna. Cabe señalar que la mayoría de las estrategias reconocidas, son percibidas como positivas por los usuarios.

Pese a ello, gran parte de los usuarios se refiere enfáticamente a las prácticas percibidas como negativas en el proceso de atención en general. Ellos sitúan estas prácticas en la dimensión atención y tienen que ver con el trato inadecuado generado por parte de algunos funcionarios, la incomodidad por los excesivos tiempos de espera en la atención en el CESFAM y también en las horas de especialidad. Así, también destaca la escasa información recibida sobre los cambios desarrollados en el establecimiento y la inadecuada infraestructura del CESFAM (aunque esto último entendido como un tema coyuntural). Así, para los usuarios entrevistados estos temas resultan en la práctica aspectos muy importantes, pues los consideran como pilares fundamentales de una atención eficiente y digna.

La referencia que se hace a las condiciones o características negativas del sistema de atención, no es menor, pues constituyen los temas visibles para que los usuarios tengan o no una buena percepción sobre el quehacer del CESFAM. Esta percepción termina por empañar, invisibilizar o disminuir el interés por las estrategias incorporadas en el proceso de transformación. Por tanto, aunque exista reconocimiento de pequeńos cambios positivos, en general ellos no dan cuenta de cambios trascendentales en las dimensiones planteadas, algunos de ellos incluso señalan que no ha habido cambios, que todo sigue "igual", incluso "peor".

Para el caso de los funcionarios, se puede señalar algo similar, es decir, ninguno de ellos reconoció cambios trascendentales en el proceso de transformación; esto 
quiere decir, que exceptuando algunas acciones, según este grupo de entrevistados, el trabajo sigue desarrollándose en la misma manera o muy similar a la anterior, es decir, antes del año 2006, en la etapa de consultorio. Las excepciones para los funcionarios se reflejan en los cambios "parciales" en las dimensiones atención, organización, participación y pertinencia cultural, pues se han incorporado algunas líneas nuevas de trabajo con las familias y comunidades. Estos cambios se consideran parciales, porque para la dimensión participación y atención en algunas postas ya se había implementado con anterioridad. Cabe señalar, que la percepción de cambios parciales por parte de los funcionarios, se apreciaron con mayor intensidad en los discursos de los profesionales, sobre todo de quienes han participado desde los inicios, creando, diseñando o tomando decisiones relevantes en cuanto a la implementación de acciones. Por su parte, los técnicos paramédicos y administrativos entrevistados notan un nivel de cambio inferior, es decir, creen que hay cambios que son mínimos, siendo los más destacados el proceso de sectorización y la utilización de algunas herramientas como el tarjetón familiar y las educaciones en las escuelas.

Ahora bien, los cambios mínimos, en donde coinciden todos los funcionarios, corresponden a la intersectorialidad. Con excepción del trabajo con las escuelas, no existe una constante, aun cuando está en el discurso de las instituciones realizar un trabajo mancomunado a favor de una mejor salud para Tirúa.

A la luz de estas perspectivas y visiones se pueden dilucidar algunos elementos explicativos como la insuficiente información que circula del proceso, a las experiencias negativas personales al respecto y/o la insuficiente infraestructura y recursos humanos. Sin embargo, no existe un marco explicativo que clarifique a cabalidad esta situación, por tanto, se avala la necesidad de realizar investigaciones cualitativas y específicamente alguna dirigida a identificar los elementos que influyen en tales percepciones.

Lo anterior, permite entrar de plano a la segunda hipótesis planteada en la investigación que señala que "existe disonancia entre la percepción de funcionarios y usuarios sobre el proceso de transformación a CESFAM en la comuna de Tirúa”, la cual es absolutamente coincidente con el trabajo de campo, pues pese a que existen algunas similitudes, se confirman grandes diferencias entre ambos grupos. Incluso, es interesante constatar también diferencias significativas al interior de 
cada uno de ellos. Así, las principales diferencias se aprecian en la dimensión organización, pues la mayor parte de las actividades realizadas en el CESFAM son percibidas por los funcionarios, pero mínimamente por los usuarios. Esto ocurre en primer lugar, porque el proceso de cambio tiene que ver con acciones internas propias del equipo, planificaciones, reuniones técnicas, informes y/o protocolos, que no son necesariamente informadas a la comunidad. En el caso de las actividades visibles como las visitas domiciliarias, por ejemplo, son percibidas por los usuarios como actividades antiguas y por tanto, no constituyen algo nuevo, por ello sólo destaca el trabajo con las escuelas.

Otra razón, es que las acciones de difusión de los cambios y actividades emprendidas por los equipos no son suficientes para que la población usuaria se sienta completamente informada. Del mismo modo, los métodos utilizados parecieran no resultar tan efectivos para una población que está en constante movimiento diario, e incluso hay una parte de esta población que no concurre nunca al establecimiento. Por tanto, quienes perciben mayores cambios, por ejemplo, en cuanto la sectorización, no lo perciben dentro de un proceso más amplio, sino como cambios aislados. Esto se puede reflejar en la comprensión del significado del nombre asignado al CESFAM: "Centro de Salud Familiar con pertinencia cultural" es una designación poco comprendida por los usuarios en comparación a los funcionarios. No existe una asociación directa entre el nombre y las actividades realizadas por el establecimiento y las ideas que destacan están siempre asociadas a nueva infraestructura.

Ahora bien, como ya se anunció más arriba, este proceso de diferencias en la percepción y conocimiento del proceso de transformación, no sólo se desarrolla entre funcionarios y usuarios, sino también se dan diferencias entre los mismos funcionarios y también entre los mismos usuarios. Así, esta misma dimensión, es evidente que quienes tienen mayor conocimiento, manejan y llevan a cabo las directrices del modelo y el proceso de transformación son los profesionales; los técnicos paramédicos manejan información a grandes rasgos y logran desarrollar las actividades que se emprenden, pero no participan en la toma de decisiones principales, los funcionarios administrativos son aquellos que menos información tienen del proceso de transformación a CESFAM, incluso les cuesta comprender qué implica y significa el modelo de salud integral. Esta situación deja al descubierto que un grupo minoritario expresado en el equipo gestor o en el grupo de 
profesionales, tiene la capacidad de decisión y organización de gran parte del proceso de transformación e implementación del modelo. La exclusión de la mayor parte de los funcionarios genera desinformación y percepción de marginación (incluso voluntaria), lo que influye en el trabajo en equipo y en el clima laboral. Por ese motivo, la percepción de las acciones tiene una perspectiva distinta según el estamento entrevistado.

En cuanto a los usuarios, también se pueden apreciar diferencias, es decir, quienes poseen mayor conocimiento e información del proceso son los dirigentes (pero aun así, en menor medida que los funcionarios), en segundo lugar a quienes se les ha realizado un estudio de familia y quienes están más alejados y acuden esporádicamente tienen menor conocimiento e información. Otra diferencia en la dimensión organizacional es el proceso de sectorización, ya que el grupo de funcionarios conoce y comprende el sentido de esta división, pero la mayor parte de los usuarios no logra identificarla, a excepción del sector centro, en donde le fue asignado un color particular para la atención médica.

En cuanto a la dimensión atención, las principales diferencias que se aprecian entre funcionarios y usuarios en el ámbito de la atención, tienen que ver con aquellos temas más relevantes. Así, mientras que para los usuarios los principales temas de atención son la eficacia, la resolutividad de la atención y el trato recibido, para los funcionarios es la sobredemanda asistencial, los recursos para el trabajo y la estabilidad-rotación laboral. También, hay una clara diferencia respecto de la percepción de las actividades de terreno, promoción y prevención. Mientras los funcionarios señalan claramente cuáles son sus actividades, cuándo y en qué circunstancias las realizan, los usuarios no están al tanto de ellas y aquellos que tienen alguna idea no conocen, ni manejan toda la información al respecto.

Los funcionarios presentan claros disensos a la dimensión atención en torno a la ejecución de las distintas acciones, esto porque algunos se encuentran a favor y otros se ven más reacios. Queda de manifiesto que la denominada "resistencia al cambio" por parte de algunos funcionarios, no tiene que ver con un discurso de resistencia al modelo en sí, sino más bien a las actividades, a la forma en que estas se planifican y desarrollan. Esto tiene que ver con dos motivos, el primero es que muchos de los funcionarios más antiguos señalan que algunas de las estrategias incorporadas son actividades que ellos han desarrollado siempre y no incorporan 
elementos nuevos; por otro lado, otras actividades no agradan, pues en su mayoría son sentidas como asignadas más que consensuadas.

En cuanto a la dimensión pertinencia cultural, se dejan ver otras diferencias. En primer lugar, entre el grupo de funcionarios, se observa también un grupo pequeño que lidera las acciones, mientras otros participan sólo de actividades masivas, además existe desinformación y distintas valoraciones al sistema de derivación. Por tanto, si bien se presentan avances en la implementación de estrategias interculturales no se desarrollan de manera transversal y constante por todos los funcionarios, sino más bien obedecen a un programa específico.

En el caso de los usuarios también existen diferencias. Cabe señalar que todos los entrevistados conocen a agentes de salud mapuches y no mapuches dedicados a resolver ciertas enfermedades y han acudido históricamente a ellos, pero la gran mayoría de ellos no está informada del sistema de derivación del CESFAM y por tanto, son pocos quienes lo han utilizado. En este contexto, las valoraciones hacia este sistema de derivación tiene distintos énfasis en los entrevistados, pues algunos lo consideran como una estrategia útil y positiva, otros sienten que su enfermedad no tuvo solución, la cual no difiere en demasía de la evaluación que hacen de otros agentes de salud, pues también depende del logro de su sanidad.

Por otra parte, es necesario señalar, como ya se había anunciado, la existencia de similitudes en las percepciones entre usuarios y funcionarios. La primera es en el ámbito organizacional, ya que ambos grupos reconocen la influencia política (alcaldía, parlamento y presidencia) en algunas dificultades del proceso de transformación. La segunda tiene que ver con la sensación de abandono, aislamiento geográfico, falta de recursos, pobreza y en general problemas de índole social. Relacionado con ello, es que ambos grupos reconocen la falta de recursos materiales e infraestructura inadecuada para el CESFAM. Aunque este tema resulta de un problema inesperado y puntual (cese de las obras) afecta profundamente el desarrollo del quehacer en el establecimiento y para la continuidad de los avances.

En cuanto al tema del trabajo intersectorial existe también coincidencia entre funcionarios y usuarios en el sentido de que ambos grupos reconocen que el trabajo ha sido incipiente y débil, destacándose en ambos casos sólo el trabajo con las escuelas. No se aprecia un trabajo fuerte con las demás instituciones al respecto, sino más bien temas aislados. Pareciera ser que el aislamiento institucional es 
una práctica instalada y finalmente cada una de ellas vela por el cumplimiento de sus propias metas y objetivos. En el caso del CESFAM la razón más evidente pareciera ser la falta de tiempo, así como la sobreexigencia del sistema de atención.

Respecto a la dimensión participación en salud, existen también más coincidencias que disensos, puesto que no existe información, tanto para usuarios como funcionarios, de todas las líneas estratégicas que este ámbito implica. En segundo lugar, existe consenso sobre algunos avances, pero el nivel de participación no es el ideal, pues los usuarios no toman decisiones importantes en temas relevantes, sino más es de carácter informativo y consultivo. Así también, la participación en las organizaciones de salud, consejos de desarrollo y comité de posta (excepción de Loncotripay y Quilantahue) es baja y por lo general no existen eficientes mecanismos de traspaso de la información de manera eficaz hacia las bases y a la comunidad. Si bien se realizan diagnósticos participativos, se hacen con un número pequeño de integrantes; muchos de ellos son dirigentes, los cuales no siempre cuentan con el respaldo de sus socios.

Por otro lado, el trabajo con las comunidades mapuches en materia de salud ha sido complejo, pues en sectores con alta población mapuche el trabajo ha sido más lento y dificultoso. La razón pareciera estar en que la figura de organizaciones propuestas, es decir, "consejos de desarrollo" y "comité de posta”, no son del todo pertinentes en su estructura organizacional. Sin duda, este tema tiene también otras aristas que no son motivo de esta investigación. Esto no quiere decir que los consejos y comités no realicen actividades, las realizan y por cierto han sido bastante destacadas. Lo que se pone en cuestión es el concepto de pertinencia y participación social que ejercen las comunidades en la gestión del CESFAM en torno a sus propias necesidades y contexto. En este sentido, por ejemplo, el sistema de reclamos, aunque es un sistema de control y detección por parte de los usuarios, resulta ineficaz, ya que en la mayoría de los casos no se recibe respuesta favorable a éstos. Así, tanto usuarios como funcionarios vinculados a este tema, creen que si bien se realiza el protocolo y plazos establecidos no presenta soluciones reales a los reclamos que se presentan.

En síntesis, posible señalar, que aunque existen coincidencias en algunos temas entre funcionarios y usuarios, la segunda hipótesis se confirma, pues existen notorias diferencias entre las percepciones de los grupos entrevistados y no sólo entre ellos, 
sino también entre los mismos funcionarios y entre los mismos usuarios. Para ambos casos, la percepción respecto del proceso de transformación está determinada por el nivel de participación e involucramiento de la persona entrevistada en él.

\section{ESTILO DE POLÍTICA PÚBLICA}

La política de salud se categorizó en base a los diferentes estilos propuestos por Blázquez \& Morata (2005) estilo Top-Down, Buttom Up y un tercer modelo que combina ambos estilos. Sin embargo, tanto usuarios como funcionarios reconocen como preponderante sólo el estilo top-down en el proceso de ejecución de las acciones implementadas en el CESFAM como se presenta en el cuadro 3.

\section{Cuadro de análisis 3: Estilo política pública}

\begin{tabular}{|c|c|c|}
\hline Estilo política pública & $\begin{array}{l}\text { Percepción } \\
\text { Funcionarios }\end{array}$ & Percepción Usuarios \\
\hline $\begin{array}{l}\text { Estilo Top Down } \\
1^{\text {a } \text { generación }}\end{array}$ & Sí & Sí \\
\hline $\begin{array}{l}\text { Estilo Buttom Up } \\
\text { 2a generación }\end{array}$ & No & No \\
\hline $\begin{array}{l}\text { Ambos estilos } \\
3^{\text {a }} \text { generación }\end{array}$ & No & No \\
\hline
\end{tabular}

Fuente elaboración propia.

El detalle presentado en el cuadro de análisis No 3, permite confirmar la tercera hipótesis que plantea que "El modelo de política pública obedece al estilo "Top Down", pues no existe retroalimentación directa entre las etapas de formulación e implementación. Esto se fundamenta, en primer lugar, en que las directrices se lanzan desde el MINSAL y se implementa en el espacio local de manera vertical y unidireccional y no circula la información desde lo local hacia el nivel central de la misma manera. En otras palabras, existe un escaso vínculo entre la formulación de la política y su realización, lo que no permite su adecuada redefinición.

Si bien a nivel local el equipo de salud y algún sector de la comunidad han podido re-organizar y/o re-plantear estas directrices con constantes innovaciones, creaciones y adaptaciones a la realidad comunal, este proceso de análisis no llega 
a enriquecer la instancia de reformulación, sino que se queda en el mismo espacio local (el equipo de salud) y en cierta medida a nivel provincial (Servicio de Salud Arauco). Esto se refuerza además con la percepción de los sujetos participantes de la investigación, ya que tanto funcionarios como usuarios sienten que las estrategias son dirigidas desde "arriba". Los funcionarios sienten que todas las directrices emanan desde el MINSAL y son operadas por el Servicio de Salud Arauco en similar lógica. Esta dinámica se proyecta al interior del CESFAM y se refleja en la relación jerárquica entre funcionarios, por ejemplo en el poder de decisión del equipo gestor (aunque en el proceso de sectorización y reuniones de equipo se ha revertido en alguna medida).

Por su parte, los usuarios, perciben que las pautas son marcadas por los funcionarios y también a nivel provincial en algunos casos. Esta situación se reconoce en todas las dimensiones planteadas, a excepción de participación en salud, en donde los dirigentes y participantes tienen más libertad de acción sobre todo con respecto al ámbito de la gestión. Por tanto, se puede concluir que, independiente de las buenas intenciones del MINSAL de implementar estrategias tendientes a mejorar la salud de la población, es una política de salud que es impuesta desde "arriba" en las etapas y espacios de su ejecución y por tanto, la hace impertinente en ciertos aspectos y momentos.

\section{RECONOCIMIENTO Y RELACIÓN DE MODELOS MÉDICOS}

La categorización de los modelos médicos se realizó en base a tres tipos: biomedicina, medicina indígena y popular y autoatención. De esta clasificación, los usuarios reconocen en sus discursos el uso de todos los modelos en sus prácticas de salud, mientras que los funcionarios sólo los dos primeros. En cuanto a la relación entre estos modelos, ambos grupos reconocen que la biomedicina es preponderante y hegemónica en el quehacer del CESFAM, sin embargo, queda de manifiesto la significativa adherencia de los usuarios a la medicina indígena popular y a la autoatención fuera del establecimiento. A continuación, un detalle de esta categorización: 


\section{Cuadro de análisis 4: Reconocimiento de modelos médicos}

\begin{tabular}{|c|c|c|}
\hline Reconocimiento de modelos & Funcionarios & Usuarios \\
\hline Biomédico & $\begin{array}{c}\text { Sí }(+++) \\
\text { (Modelo integral, salud } \\
\text { familiar, salud intercultural) }\end{array}$ & Sí (+++) \\
\hline Medicina indígena y popular & Sí $(+)$ & Sí $(++)$ \\
\hline Autoatención & No $(-)$ & Sí (++) \\
\hline
\end{tabular}

Fuente: elaboración propia.

A través de este cuadro, queda de manifiesto que la hipótesis principal referida a que "el proceso de transformación que ha vivido el CESFAM I.J.R. de Tirúa, que incluye enfoques de salud integrales con enfoque familiar, comunitario y con pertinencia cultural se da en una situación de tensión y confrontación en el quehacer dada por la subyacente hegemonía del modelo médico por sobre los otros enfoques y formas de atención" se confirma a partir de los resultados de la investigación.

Para el caso de los funcionarios, el modelo más reconocido, aceptado y practicado en el quehacer cotidiano de la salud es el modelo biomédico. Dentro de este contexto, el grupo de funcionarios también reconoce otros enfoques derivados como la salud familiar y la salud intercultural, que intentan un abordaje más integral del proceso de salud-enfermedad, pero que finalmente toman un rol secundario al modelo biomédico. Por otra parte, algunos de los funcionarios advierten la presencia de elementos de la medicina mapuche en las consultas, enfermedades y tratamiento de algunos usuarios y conocen a grandes rasgos el sistema de derivación a machi, componedor y partera implementado en el CESFAM, sin embargo, no se deja ver en sus discursos (con algunas excepciones) un conocimiento formal y profundo respecto de estos modelos médicos a los que adhieren los usuarios y las prácticas que se generan en torno a ellos.

Pese a lo anterior, este grupo de entrevistados reconoce la hegemonía del modelo biomédico. Entre los factores que posibilitan esta hegemonía se encuentra la formación académica, la cual ha estado enfocada primordialmente a la biomedicina y por tanto, sus conocimientos de otros modelos y enfoques de salud, son desconocidos o mínimos y a la vez subalternos. Si bien en los últimos años los funcionarios 
han recibido una mayor cantidad de capacitaciones en estos enfoques, muchos de ellos refieren mayor dominio y apertura por opción personal.

Otro factor explicativo es que el modelo biomédico se reproduce y potencia en el quehacer mismo de la atención. Esto significa que los funcionarios tengan o no formación en enfoques de salud integral, se insertan en un mundo laboral sustentado en la biomedicina. Esta idea se refleja en el discurso de los funcionarios más antiguos, quienes declaran que este modelo ha sido por décadas el modelo validado en los establecimientos de salud y por tanto, el que se ejerce de manera normal y obligatoria. Esta validación se ha realizado por parte del Estado a través del MINSAL, el cual exige una forma de operar a través de programas de salud, metas cuantitativas y un estilo de relación de jerarquía entre funcionarios y también con los usuarios. Es por ello que las estrategias de salud englobadas en el "Modelo de atención integral con enfoque familiar y comunitario", se enfrentan en la práctica a una fuerte presión en su ejecución, puesto que las actividades biomédicas se encuentran profundamente arraigadas y terminan por ocupar un lugar preponderante. La mayoría de los funcionarios cree que existen limitantes para poder llevarlas a cabo a excepción del trabajo en el CECOSF de Quidico, en donde los propios funcionarios perciben un trabajo con familias y comunitario más avanzado.

Ahora bien, para el caso de los usuarios, se puede señalar que ellos también reconocen la presencia del modelo biomédico en tanto observan y conocen las prácticas médicas realizadas por el CESFAM, de igual modo distinguen qué tipo de enfermedades o en qué situaciones deben recurrir y solicitar asistencia; esta concurrencia se relaciona principalmente con las consultas de morbilidad, de urgencia y controles de salud.

Además de lo anterior, queda de manifiesto un hallazgo relevante y es que los usuarios adscriben a la medicina tradicional y al modelo de autoatención de manera recurrente y significativa. Con respecto a prácticas basadas en la medicina tradicional aparece la concurrencia a machi, así como al componedor de huesos, partera, naturistas, iriólogo, gitanos y también las iglesias evangélicas. Por su parte, el modelo de autoatención se expresa principalmente en que los usuarios modifican, suspenden o complementan el tratamiento biomédico y también hacen uso de otros recursos, en su mayoría hierbas medicinales y en menor medida en otros recursos de conocimiento comunitario trasmitido de generación en generación. Es 
importante mencionar que la totalidad de los usuarios entrevistados que hacen uso de hierbas medicinales reconocen un resultado positivo ante su uso. Así, es posible señalar que los usuarios utilizan ambos modelos médicos (tradicional y autoatención) de manera excluyente, complementaria, previa o posterior al modelo biomédico. Estas prácticas están establecidas y son conocidas de tiempos ancestrales, mientras que otras son más recientes, lo cierto es que son en la práctica modelos absolutamente vigentes y muchas veces el modelo principal dependiendo de la importancia que le dan los usuarios.

Ahora bien, con respecto a la hegemonía de estos modelos, los usuarios también reconocen que el modelo biomédico es predominante dentro del quehacer del CESFAM. Los factores más evidentes resultan ser la considerable demanda biomédica, en tanto es eficiente a la enfermedad o consulta del usuario, y la condición de medicina oficial y objeto de la política pública para toda la población. Por tanto, las críticas que derivan de este modelo por parte de los usuarios, apuntan a los aspectos que no son resultado de una buena atención resolutiva y eficaz y no a la concepción de la biomedicina en sí. Lo anterior también se refleja en la relación que se establece entre funcionarios y usuarios. Según estos últimos y pese a que muchos de ellos reconocen una relación muy cordial con los primeros, se da una vinculación de "experto-inexperto" con un evidente ejercicio de poder del profesional sobre el diagnóstico y el tratamiento. En este sentido, una parte de los entrevistados siente que no son tratados horizontalmente, sino más bien en función de un status superior-inferior.

Es interesante reflexionar que aunque el modelo biomédico es oficial y más visible en el contexto del CESFAM, fuera de éste, las personas dan paso a sus conocimientos populares y tradicionales para lograr el proceso de sanación de su respectiva enfermedad. Así, estos acuden a la biomedicina cuando efectivamente lo encuentran necesario y/o cuando el problema no pudo ser resuelto. A raíz de ello, se da una especie de vacío bidireccional en la implementación del modelo de atención integral con enfoque familiar y comunitario, en primer lugar porque en la práctica no se incorporan los modelos médicos en el quehacer del CESFAM en toda su complejidad, y en segundo lugar porque los usuarios no reconocen, ni valoran todas las estrategias de salud integrales incorporadas a la luz del proceso de transformación; en otras palabras, ni funcionarios ni usuarios comprenden a cabalidad los respectivos modelos y/o enfoques de salud. 
Si bien las estrategias de salud intercultural en el CESFAM están enfocadas al trabajo con machi, componedor de huesos y parteras principalmente, lo que constituye un avance importante, queda de manifiesto que las prácticas de salud de los usuarios se desarrollan en un abanico mucho más amplio en cuanto a los diagnósticos, tratamientos y agentes de salud. Los usuarios tienen un sinnúmero de creencias basadas en el sentido popular e indígena, las que determinan la explicación de la enfermedad y por consecuencia la elección de su respectivo tratamiento. En este sentido es importante señalar que muchas de las enfermedades relatadas por los usuarios se enmarcan en el plano espiritual y/o religioso por lo que el tratamiento se desarrolla paralela o únicamente en este plano. Destacan en los relatos las actividades encomendadas a las iglesias (principalmente evangélicas) y las "limpiezas" de sus casas. Por tal motivo, los usuarios desarrollan el ciclo del enfermo ${ }^{1}$ de acuerdo a sus creencias y experiencias previas al respecto. Pueden recurrir a agentes de medicina popular en primer lugar y continuar con un circuito de sanación en el contexto comunitario, para finalmente llegar al sistema de atención en el CESFAM o viceversa. Lo relevante es que cada usuario toma decisiones y opta por alternativas de solución que muchas veces son desconocidas para el equipo de salud, aunque se pueden apreciar algunas excepciones dependiendo de la cercanía y confianza con el funcionario(a).

De esta manera, se permite aseverar que tanto los modelos, experiencias, conocimientos y valoración de las prácticas de salud son distintas entre ambos grupos entrevistados. Esta idea pareciera encontrar un razonamiento obvio, sin embargo, es interesante destacarlo porque se puede apreciar en todas las dimensiones abordadas en esta investigación.

\section{CONCLUSIONES}

De los resultados y análisis obtenidos en función de los objetivos de esta investigación se pueden dilucidar las siguientes conclusiones. En cuanto al objetivo específico relacionado con describir el proceso de transformación de consultorio a centro de salud familiar que propone el Ministerio de Salud para la atención primaria,

1 Es el camino que realizan los enfermos y/o sus familias en el proceso de salud - enfermedad - atención. 
realizado a partir de la revisión de documentos formales, se puede concluir que éstos dejan ver una reflexión sobre las falencias del modelo biomédico y la intención de instalar paulatinamente enfoques de atención en salud más integrales y pertinentes a las realidades sociales. Además, existen evidencias documentales de la puesta en marcha de acciones en base al "modelo de salud integral con enfoque familiar”, estrategias de salud intercultural y estrategias de participación social, las cuales comenzaron a llegar al CESFAM I.J.R. a través de distintos programas con instructivos, capacitaciones y reuniones de coordinación para funcionarios.

Pese a lo anterior, se constatan debilidades desde el comienzo en su aplicación, pues muchos de los documentos guía que se crearon en la década del noventa entregan orientación general, pero escasos lineamientos técnicos específicos que indiquen cómo llevar a cabo las acciones. En este sentido, los manuales, sistematizaciones e investigaciones aparecen en ańos posteriores y en muchos casos, estos documentos no estuvieron disponibles en el CESFAM como insumo de trabajo. En este contexto, las directrices del proceso de transformación y documentos que las contienen recaen directamente en los funcionarios. Si bien el objetivo de cambio involucra necesariamente a la comunidad, la responsabilidad inicial de implementar y liderar las acciones está dirigida al equipo de salud, lo que evidentemente coloca a la comunidad en un lugar receptivo y menos protagónico.

En relación con lo anterior y en base al objetivo específico No 2, que propone describir y analizar el proceso de implementación del CESFAM a partir de la percepción de usuarios y funcionarios, es posible concluir que este proceso se ha desarrollado en forma lenta, con acciones iniciales de sensibilización desde el año 2003 y estrategias de cambio a partir del año 2006. En general estas acciones de cambio han permitido certificar al establecimiento como CESFAM y se han realizado en el ámbito de la atención, organización, pertinencia cultural, participación e intersectorialidad, pero con distintos énfasis y avances cada uno.

Las percepciones respecto de estas estrategias y cambios que han provocado en el sistema de atención de salud están divididas entre funcionarios y usuarios, es decir, se aprecian diferencias entre ambos grupos y también al interior de cada uno de ellos; en otras palabras, algunos identifican ciertos cambios, otros sólo en algunas dimensiones y otros no identifican ninguna acción que intente transformar el quehacer del CESFAM. 
De esta manera, figuran usuarios que tienen una mirada positiva de los cambios, sin embargo, gran parte de ellos tiene una apreciación bastante crítica con respecto al sistema de atención, lo que ha hecho, por una parte, invisibilizar los logros y avances y por otro, relegarlos a un nivel secundario. Las mayores disconformidades se dan en la dimensión atención y tienen que ver con recibir trato inadecuado y jerárquico, bajo nivel de resolutividad en las atenciones de especialidad y largas esperas diarias en las consultas (urgencias y morbilidad) y controles, escaso flujo y claridad de la información relevante, escasez de recursos primordiales (medicamentos, ambulancias, infraestructura adecuada, recurso humano, entre otros). Esta situación genera experiencias negativas personales y/o familiares ante problemas de salud no resueltos, y como consecuencia, lejanía en actividades que no tienen que ver con lo asistencial propiamente tal.

Los funcionarios en tanto, reconocen que la principal problemática en el ámbito atención corresponde a la gran demanda asistencial que enfrenta el CESFAM, pues con los recursos materiales y humanos disponibles no es posible cubrir de manera satisfactoria, ni destinar mayor tiempo a otras actividades anexas. A diario los equipos se ven sobrepasados por los usuarios y sobre exigidos por los requerimientos de los diferentes programas de salud y metas que impulsa el Ministerio de Salud.

Por tanto, es imposible pensar que el proceso de cambio se daría de manera fácil y rápida. Tal como lo plantearon Nadler (1980) y Kisil (1984), la introducción de cualquier modificación trae consigo algunos problemas de relaciones, poder y resistencia al cambio, que para el caso de Tirúa se manifiestan principalmente en la toma de decisiones al confrontarse las visiones de los distintos funcionarios y en la ejecución de algunas actividades biomédicas versus las derivadas del "modelo salud integral con enfoque familiar y comunitario”.

Si bien las diferencias y oposición ante ciertos temas se han mantenido, la clave parece estar en lograr equilibrio del quehacer y en toma de decisiones, haciendo énfasis en las relaciones horizontales y en el trabajo en equipo en pos de un objetivo común. Ello implica integrar y velar por el desarrollo en conjunto de los establecimientos de la red local (postas, CECOSF y CESFAM), de los sectores y los diferentes estamentos, evitando competencias y diferencias notorias entre éstos.

En general, se puede concluir en función de este objetivo, que los entrevistados perciben cambios mínimos y parciales, pero en general no consideran que las 
acciones emprendidas sean trascendentales para lograr la transformación a CESFAM que propone el Ministerio de Salud. Por tanto, la incorporación de los nuevos enfoques y modelos integrales de salud familiar no son del todo distinguibles en la práctica y por tanto, no son valorados como se espera por la comunidad. Los principios sobre participación social e interculturalidad en salud se encuentran en una etapa incipiente de implementación considerando lo que en teoría el Ministerio de Salud (2008), Albó (2010) y Campos (2010) proponen para cada uno de ellos.

Pese a lo anterior, es necesario destacar que éste es un proceso en pleno desarrollo y su historia lejos de terminar se va construyendo diariamente. Sin duda, esta realidad constituye un buen marco de razones para profundizar en una futura investigación cualitativa.

En referencia al objetivo específico No 3, que plantea el discutir y analizar la pertinencia de la aplicación en el nivel local, de la política pública diseñada desde el nivel central, se pueden plantear dos grandes conclusiones. La primera es confirmar que esta política de salud está creada y orientada en base al estilo "top -down", pues como planteó Blázquez \& Morata (2005), existe una separación entre lo administrativo y la vida pública de la política y se obvia la relación entre las etapas de formulación-implementación-redefinición. Las acciones provienen del nivel central y la mayor parte de las decisiones son asumidas en una perspectiva de jerarquía que caracteriza a las instituciones de salud; esta jerarquía adopta distintos protagonistas según el contexto nacional, provincial y local y se presenta en la interacción entre funcionarios y también entre funcionarios y usuarios.

La segunda conclusión deriva de la primera y constata la limitada acción que tiene el nivel central de controlar la implementación de esta política en el nivel local, lo que hace cuestionar su pertinencia en algunos aspectos de la fase de ejecución. Este cuestionamiento confirma lo adelantado por Bello (2009) de que una intervención en salud exitosa requiere de intervenciones efectivas, recursos suficientes y un modelo de gestión que asegure una implementación adecuada, condiciones que en este estudio se ponen en duda al constatar que estos elementos no siempre están presentes de forma óptima.

Así, un primer elemento vinculado a lo anterior, dice relación con las herramientas técnicas y capacidades que tienen los equipos, los recursos disponibles, la estabilidad laboral, el clima organizacional al interior del CESFAM y también las 
capacidades, recursos, dinámica y vinculación de la comunidad local, que se desarrollan en Tirúa en un contexto especial de ventajas, pero también con obstáculos.

Otro elemento que se controla con dificultad es lo que la carta de Otawa en 1986 llamó determinantes sociales en salud. En otras palabras, elementos como el trabajo, transporte, educación, alimentación y saneamiento ambiental, sumado a la pobreza, aislamiento geográfico y ruralidad, para Tirúa son claves en la implementación de las acciones impulsadas en este proceso de transformación. Así también lo es el contexto cultural, la presencia de la población mapuche y no mapuche y sus respectivas dinámicas, costumbres y cosmovisión han establecido una propia lógica en torno al proceso de salud-enfermedad y en las formas de atención.

También figuran los bajos niveles de participación social en la comuna de Tirúa, las estrategias de participación instauradas desde la institucionalidad en general se presentan desgastadas y los usuarios han adoptado acciones de "no participación" o "participar a su manera", pues las formas propuestas en el caso de la salud no siempre se ajustan a las necesidades y mecanismos de las comunidades y organizaciones existentes.

A partir de lo anterior, ha surgido un contexto de facilitadores y obstaculizadores determinantes en la puesta en práctica de las acciones encomendadas, por tanto, no es pertinente aplicar de manera lineal las directrices establecidas. Los funcionarios son los intermediarios y encargados de llevar a cabo estas directrices y exigencias, pero al encontrarse con los impedimentos del contexto local y con las altas exigencias ministeriales (metas sanitarias e indicadores de desempeño) están obligados a crear, adaptar e innovar. Esto puede resultar muy positivo, sobre todo al considerar las destacadas capacidades locales; no obstante, la experiencia sigue demostrando la lógica "top down".

Esta realidad debe ser comprendida, considerada y replanteada por el Ministerio y Servicios de Salud, pues como plantea Molina \& Cabrera (2008), el Estado es el responsable de dar respuesta a los problemas públicos, pero debe hacerlo de manera articulada con un número amplio de actores sociales y velar por una activa participación ciudadana. Esto coincide con Blázquez \& Morata (2005), pues debieran aplicar para el éxito en la política pública, ni el estilo "top-down" ni "buttom-up" por sí solos, sino que una mezcla de ambos en el proceso de implementación. 
En base a todo lo expuesto y en virtud del objetivo general que propuso describir, analizar y discutir el proceso de transformación para atención primaria en el CESFAM I.J.R. de Tirúa, Región del Biobío, entre los años 2006 y 2011, se puede concluir que se han realizado acciones a favor de este proceso que reflejan un "modelo" de salud caracterizado por una mezcla de lo biomédico con pequeños matices que integran lo biopsicosocial, el enfoque familiar, enfoque intercultural (aplicado a la medicina mapuche). Además, se constata la coexistencia de otros modelos médicos en las prácticas de los usuarios, adscritas a medicinas populares, indígenas y de autoatención, con una fuerte presencia de la dimensión espiritual y/o religiosa, lo cual se sitúa en plena concordancia con la clasificación de Menéndez (1982) y Citarella (2010) sobre la existencia de tres modelos médicos: Modelo Médico Hegemónico (biomedicina), Modelo Médico Alternativo Subordinado (tradicionales) y el Modelo Médico de Autoatención (de propio usuario).

De lo anterior, se desprenden dos ideas concluyentes, la primera tiene que ver con que los enfoques de salud implementados por el CESFAM de Tirúa resultan parciales e incompletos en la comprensión del proceso de salud-enfermedad de la población y en algunas estrategias de atención. Esto no quiere decir en ningún caso que el CESFAM debe hacerse cargo de ello, más bien reconocerlas, considerarlas y respetarlas en el ciclo de atención del usuario(a).

La segunda idea concluye la existencia de una confrontación y hegemonía del modelo biomédico por sobre las otras formas de atención, enfoques de salud y modelos existentes, donde el primero tiende a establecer una relación de hegemonía - subalternidad. Esta relación se expresa en todas las pautas, costumbres, protocolos, jerarquías, metas y evaluaciones presentes en el quehacer del CESFAM, pues tienden a sobrevalorar lo biomédico. Por esta razón y pese a que se han incorporado estrategias de salud más integrales en este proceso de cambio, toman un rol secundario y alternativo.

\section{ReFERENCIAS BibliográficAS}

Albó, X. (2010). “Interculturalidad, Estado y Pueblos Indígenas”. En Campos, Citarella \& Zangari (editores). Yanchay Tinkuy: Salud Intercultural en Bolivia y América Latina. Bolivia: Gente Común. 
Bello, D. (2009). La Participación en Politicas de Atención Primaria de salud: Estudio de Caso en La Florida y Peñalolén. Tesis para optar al grado de Magíster en Gestión y Políticas Públicas. Universidad de Chile. Santiago de Chile.

Blázquez, B. \& Morata, B. (2005). "La Implementación de las Políticas Públicas: Modelos Analíticos y el Papel de la Administración Pública”. En Pérez, M. (editor). Análisis de las Políticas Públicas. España: Universidad de Granada.

Campos, R. (2010). “Una Visión General sobre la Salud Intercultural en Pueblos Originarios”. En Campos, Citarella \& Zangari (editores). Yanchay Tinkuy: Salud Intercultural en Bolivia y América Latina. Bolivia: Gente Común.

Citarella, L. (2010). "Algunas reflexiones sobre Modelos y Sistemas Médicos”. En Campos, Citarella \& Zangari (editores). Yanchay Tinkuy: Salud Intercultural en Bolivia y América Latina. Bolivia: Gente Común.

Cuevas, K.; López, P.; Mancilla, C. (2006). Coexistencia de los Enfoques Biomédico y Biopsicosocial en los Discursos de los Profesionales de la Atención Primaria en Salud. Tesis para optar al grado de Magíster en Salud Pública mención Gestión Primaria. Universidad de Valparaíso. Valparaíso. Chile.

Corbetta, P. (2007). Metodología y Técnicas de Investigación Social. España: Mc Graw Hill.

Lahera E. (2002). Introducción a las Políticas Públicas. Chile: Fondo de Cultura Económica.

Menéndez, E. (2010). "Modelos, Saberes y Formas de Atención de los Padecimientos: de Exclusiones Ideológicas y de Articulaciones y prácticas”. En Campos, Citarella \& Zangari (editores). Yanchay Tinkuy: Salud Intercultural en Bolivia y América Latina. Bolivia: Gente Común.

Ministerio de Salud (1997). De Consultorio a Centro de Salud. Santiago: Impresos La Nación.

Ministerio de Salud (2005). Modelo de Atención Integral en Salud. Serie Cuadernos Modelo de Atención No 1. Santiago: Subsecretaría de Redes Asistenciales.

Ministerio de Salud (2006). Politica de Salud y Pueblos Indigenas. Chile: MINSAL/FONASA.

Ministerio de Salud (2008a). Manual de Apoyo a la Implementación del Modelo de Atención Integral con Enfoque Familiar y Comunitario en Establecimientos de Atención Primaria. Santiago: Subsecretaría de Redes Asistenciales.

Ministerio de Salud (2008b). En el Camino a Centro de Salud Familiar. Santiago: Subsecretaría de Redes Asistenciales.

Molina, G. \& Cabrera, G. (2008). Politicas Públicas en Salud: Aproximación a un Análisis. Colombia: Universidad de Antioquia. 
Instituto Interamericano de Derechos Humanos (2006). Campaña Educativa sobre Derechos Humanos y Derechos Indigenas: Salud Indigena y Derechos Humanos. Manual de Contenidos. Costa Rica: ASDI-OPS.

Ringeling, I. (1986). La Implementación del Enfoque familiar en el Sistema de Atención Primaria. En Florenzano, R.; Horwitz, N.; Ringeling, I.; Ventureli, G. \& Villaseca, P. (1986). Salud Familiar. Santiago: Universidad de Chile. Fundación Kellogg. Corporación de Promoción Universitaria.

Rodríguez, G.; Gil J. \& García, E. (1999). Metodología de la Investigación Cualitativa. Málaga: Ediciones Aljibe.

Ruiz, J. (2009). Metodología de la Investigación Cualitativa. Bilbao: Universidad de Deusto.

Sáez, M. (2007). “Interculturalidad en salud en Chile: de la teoría a la práctica”. En Lolas, F.; Martin, D. \& Quezada, A. (editores). Prioridades en Saludy Salud Intercultural. Chile: Santiago de Chile: Centro Interdisciplinario de Estudios de Bioética Universidad de Chile.

Tamayo, M. (1997). "El análisis de las Políticas Públicas”. En Bañon, R. \& Carrilo, E. (editores). La Nueva Administración Pública. España: Alianza Editorial.

\section{Referencias Electrónicas}

Cortés, A.; Araya, E.; Lerma, G. (2004). Centro de Salud Familiar, un cambio de modelo, desafío para los equipos de salud. Ensayo presentado en Diplomado Salud Pública y Salud familiar. Universidad Austral de Chile. Recuperado el día 24.08.2011, de: http://www.medicina. uach.cl/postgrado/diplomados/saludpublica/trabajos.php

Dirección de Vialidad (2012). Distancia entre ciudades. Recuperado el día 20.02.2012, de: http:// servicios.vialidad.cl/Distancias/Distancias.asp?Regiones=16

Drago, M. (2006). La Reforma al Sistema de Salud Chileno desde la Perspectiva de los Derechos Humanos. Serie Políticas Sociales No 121. Naciones Unidas-CEPAL. Recuperado el día 10.10.2011, en: http://www.cepal.org/publicaciones/xml/7/25787/sps121_lc12539.pdf

Menéndez, E. (1982). La Crisis del Modelo Médico y las Alternativas Auto gestionadas en Salud. Cuadernos Médicos Sociales (21). Pp. 1-12. Recuperado el día 10.10.2011, en: http://www. ilazarte.com.ar/cuadernos/pdf/n21a071.pdf

Menéndez, E. (1988). Modelo Médico Hegemónico y Atención Primaria. Trabajo presentado en las Segundas Jornadas de Atención Primaria en Salud. Buenos Aires. Recuperado el día 1.10.2011, en: http://www.chubut.gov.ar/salud/capacitacion/imagenes/eje1,\%20enc.1,\%20 Modelo\%20M\%E9dico\%20Hegem\%F3nico\%20y\%20APS,\%20E.Menendez.pdf 
Ministerio Secretaría General de Gobierno (2000). Instructivo Presidencial sobre Participación Ciudadana. Recuperado el día 24.06.2012, de: http://www.guiaweb.gob.cl/recursos/documentos/InstructivoPresidencialParticipacion.df

Organización Internacional del Trabajo (2006). Convenio 169 de la OIT sobre pueblos indígenas y tribales en países independientes. Chile. Recuperado el día 30.11. 2011, de http://www. oitchile.cl/pdf/Convenio\%20169.pdf

Organización Mundial de la Salud (1978). Atención Primaria en Salud, Alma Ata 1978. Serie salud para todos No 1. Pp. 2-21. Recuperado el día 9.9.2011, de: http://www.paho.org/spanish/ dd/pin/alma-ata_declaracion.htm

Organización Mundial de la Salud (1986). Carta de Otawa. Recuperado el día 02.12.2011, en http://www.paho.org/spanish/hpp/ottawachartersp.pdf

Organización Mundial de la Salud (2007). Declaración de las Naciones Unidas sobre los derechos de los pueblos indígenas, Resolución 61/295 Aprobada por la Asamblea General. Recuperado el día 24.06.2012, de: http://www.un.org/esa/socdev/unpfii/documents/DRIPS_es.pdf

Organización Mundial de la Salud (2008). La atención primaria en salud, más necesaria que nunca: Informe de la Salud en el Mundo. Recuperado el día 2.12.2011, de: http://www.who.int/ whr/2008/08_report_es.pdf

Seremi de Salud del Biobío. (2011). Diagnósticos comunales de salud con enfoque de Determinantes Sociales de la Salud, comuna de Tirúa. Unidad de Epidemiología, Departamento de Vigilancia y Control de Enfermedades. Recuperado el día 6.12.2011, de: http://www.seremidesaludbiobio.cl/epidemiologia/archivos/diagnosticos/tirua.pdf

Recibido: 03 septiembre 2012 / Aprobado: 20 noviembre 2012 